\title{
An Extraordinary Issue for Extraordinary Times
}

\author{
Morgan T. Sammons
}

Published online: 6 May 2020

(C) National Register of Health Service Psychologists 2020

We are publishing a rapid response section in this issue of the Journal of Health Service Psychology to acknowledge the profound influence that the coronavirus has had on our society and our profession.

Just as most practitioners have gone to an online platform to provide patient services, the National Register has shifted our business purely to teleworking. We have found in consequence that our workloads have increased. The absence of the rhythm of office life has led to work creeping into times once devoted to family and relaxation. None of us can predict when we will return to "normal," nor can we predict what behavioral patterns will persist once shelter-in-place edicts end. What we can predict is that this shift in work/life balance will have negative repercussions on individuals and families unless we impose limits on the amount of our days that we devote to our professions. The concept of the "weekend" is a relatively recent development in industrialized societies; the five and one-half day workweek standard remains in living memory for many of us. Management studies teach us the essential fact that more time spent at work does not result in greater productivity. We must ensure that the personal and societal benefits of the weekend are not a casualty of the current situation.

Although the history has become all too familiar, it is important to emphasize the speed at which these changes have engulfed us. In December 2019, healthcare workers in Wuhan, China, noted the emergence of a new pneumonia that bore close resemblance to symptoms of Severe Acute Respiratory Syndrome (SARS), a viral illness that had become a regionally limited epidemic approximately 15 years earlier. On December 31, 2019, the government of China notified the World Health Organization of the emergence of this new pneumonia. Within two weeks, WHO virologists had identified the causal agent as a novel coronavirus, in the same family as the causal virus of SARS and Middle Eastern Respiratory Syndrome, a highly lethal variant prevalent in Saudi Arabia, and named the illness COVID-19. On January 31, WHO declared a public health emergency of international concern. In the United States, the federal government was slow to respond, and such a declaration was not made for another six weeks. As I write this, there are now over 1.8 million cases of COVID-19 worldwide, with over 113,000 deaths. Almost $25 \%$ of these cases are in the United States, which accounts for almost one fifth of the deaths reported worldwide.

\section{Rapid Response Articles}

Uncertainly resulting from COVID-19 is reflected in a rapid response survey that the National Register and The Trust conducted approximately two weeks into the crisis, when many psychologists had already begun experiencing significant changes in their work. The first article reports the results of a survey of over 3,000 licensed psychologists, which found that most practitioners had rapidly transitioned to service delivery via telehealth, and that the majority had experienced a decline in caseload during the transition. The long-term ramifications of these observations cannot be known at present. We are not, however, able to predict the extent to which telepsychology becomes a part of our professional repertoire in the longterm. We have demonstrated that the technology exists and can be deployed in support of patient care, but this is not a new finding. We have encountered some of the limitations, such as the difficulty in performing new patient intakes or conducting psychological testing when many assessment instruments remain un-normed for anything other than in-person administration. What remains to be seen is the extent to which patients and providers are willing to substitute distance service provision for face-toface treatment. While we did not survey patients directly, our findings suggested that their embrace of distance technology may be less enthusiastic than those of practitioners. We also found that many practitioners did not anticipate continuing to use telepsychology once physical distancing measures are removed. Telepsychology has established itself as a technology of convenience. The future will determine whether it becomes a wholesale 
substitute for in-person assessment. This depends, of course, not only on the attitudes of providers and patients but also on the response of regulators and insurors. At this point in time, only a minority of states have removed barriers to out-of-state practice. Those that have allowed out-of-state practice have done so on a temporary basis only, just as the federal government has suspended enforcement of HIPAA violations on a temporary basis only.

Our second rapid response article, by Dr. John Norcross and Colleen Phillips, emphasizes nine steps that psychologists and other healthcare workers must take to both protect themselves and model self-preserving behavior to others. "Why do you take valuable time to expose psychologists to selfcalming exercises" one psychologist snapped peevishly at me recently. "We are psychologists, we know how to do this!" Therein lies the lesson: Simply because we are cognitively aware of what needs to be done, we may not-indeed likely do not - practice it. Norcross and Phillips remind us to limit our intake of coronavirus news, maintain a regular work schedule, monitor our own as well as our patients' cognitive errors, and to take "mindful moments" among other self-care techniques.

Ethical decision-making is made more challenging in times of constrained resources. How do we determine which patient is eligible for potentially life-saving care, such as ventilator access? The process of triage in mass casualty work is always wrenching, in that it implies that some patients for whom heroic measures would be applied in ordinary circumstances will not receive such services. Drs. Roger Williams, Jessica Brundage, and Erin Williams present an interesting article on moral distress in times of widescale clinical emergencies as the third rapid response article. When decisions are required that counter established clinical procedure or indeed the clinician's moral compass, psychic injury can occur. The authors contextualize moral distress with examples drawn from COVID-19 end-of-life decisions. Importantly, they note that not only is the establishment of institutional policies that make decision-making processes explicit requisite in reducing the risks of moral injury, so is an institutional process for anticipating and responding to moral injuries suffered by providers acting under unusual restraints.

\section{Regular Journal Articles}

However we adapt to a physically distant world in uncertain times, an enduring reality is the obligation to continue to provide patient care. Dr. Anthony Ragusea provides us with a review of adverse drug reactions of which all psychologists, not just prescribing psychologists, should be aware. Educating patients as to the difference between an adverse drug reaction and a negative side effect is essential, particularly when providers may lack physical cues when providing services via telepsychology. Dr. Ragusea outlines adverse drug reactions associated with common classes of psychotropics and suggests management strategies.

Non-COVID related emergencies will continue to occur, and we must stand ready to provide appropriate intervention. Dr. Brad Grunert outlines the role of psychologists in treating a set of injuries not generally considered to be within the purview of mental health — industrially related hand injuries. Any physical insult may create an adverse psychological response. Hand injuries, because of their visibility and direct association with functionality, may be more likely to do so. Dr. Grunert provides specific guidance for psychologists working as a part of a hand-trauma team to address psychological threats that occur at each phase of the recovery process. By doing so, he again demonstrates a key finding in health psychology: Addressing psychological distress at appropriate intervals throughout the recovery process is an unquestionable component of complete recovery and return of functionality.

We are as a profession called upon to respond forcefully in unusual times. As noted in the analysis of our survey of psychological practices, our profession has been fundamentally shaped by national trauma. The first two world wars played a critical role in the development of psychological science and practice, and brought the need for effective psychological intervention to the attention of policy makers and the public. Other conflicts, such as Vietnam and the current conflicts in Afghanistan and Iraq have further amplified the role of mental health in resolving combat-related psychological injuries but also in shaping public response to mental illness. It is too early to determine if enduring changes to our practice and profession will result from the coronavirus emergency. But large scale societal crises have resulted in such changes in the past, and it is not unreasonable to believe that we must be prepared to deal with similar, if unknown, consequences of the crisis affecting us today.

Publisher's note Springer Nature remains neutral with regard to jurisdictional claims in published maps and institutional affiliations.

Morgan T. Sammons, PhD, ABPP, is the executive officer of the National Register of Health Service Psychologists. He is a retired Navy captain and was formerly the U.S. Navy's specialty leader for clinical psychology. 